\title{
Effects of alcohol consumption on circulating lipid in fatty liver disease and Represent Potential cardio metabolic risk markers in Kathmandu
}

\author{
Surya ram DUWAL ${ }^{12}$, Urusha MAHARJAN*1 \\ 'Department of Biochemistry, Central Diagnostic Laboratory and Research Center, Nepal \\ ${ }^{2}$ Kedarnath Medical Hall and Health Care Center, Biochemistry, Nepal
}

Introduction: liver is the principal site for formation and clearance of lipoproteins in our body. Alcohol-induced fatty liver (steatosis) was believed to result from excessive generation of reducing equivalents from ethanol metabolism, thereby increasing fat accumulation. Liver disease is the greatest cause of death related to alcohol and a major public health problem especially in developing country. High alcohol consumption is a major cause of morbidity, yet alcohol is associated with both favorable and adverse effects on cardio metabolic risk markers.

Methods: After consent, the present study examined aged $\geq 35$ years from Kathmandu between 2019 and 2020. Data on current drinking status, age, ethnic group, educational background, waist circumference, body mass index, blood pressure, and lipid profile was measured. Baseline venous blood sample was collected aseptically according to World Health Organization (WHO) guidelines with various inclusion and exclusion criteria. Processing was done in central diagnostic laboratory and research center.

Results: Out of 780 patients $17 \%$ were female and $83 \%$ were male. Mean \pm standard deviation of age, bmi, waist circumference, hipcircumference, waisthipratio, blood pressure were $45.93 \pm 10.92,29.41 \pm 2.98,100.97 \pm 7.0,105.94 \pm 6.5,0.94 \pm 0.1$, and $132 \pm 12 / 83 \pm 17$ respectively. TG/HDL-C ratio, LDL-C/HDL-C ratio, TG, cholesterol, and lipid accumulation product significantly higher in drinkers than normal reference range. Majority were ethnic group with low socioeconomic and literate background.

Conclusions: Abnormalities in lipid profile are correlated with alcohol consumption, which calls for intervention strategies to prevent dyslipidemia and control risk factors for cardiovascular disease. Alcohol consumption is associated with a complex metabolic signature, including aberrations in multiple biomarkers for elevated cardio metabolic risk factors. 